\title{
THE ALGEBRAIC $K$-THEORY OF OPERATOR ALGEBRAS
}

\author{
JONATHAN ROSENBERG
}

April, 1996

Dedicated to the memory of Robert Thomason

\begin{abstract}
We the study the algebraic $K$-theory of $C^{*}$-algebras, forgetting the topology. The main results include a proof that commutative $C^{*}$-algebras are $K$-regular in all degrees (that is, all their $N^{r} K_{i}$-groups vanish) and extensions of the Fischer-Prasolov Theorem comparing algebraic and topological $K$-theory with finite coefficients.
\end{abstract}

\section{INTRODUCTION}

This paper is concerned with the algebraic $K$-theory of $C^{*}$-algebras, that is, when they are considered purely algebraically as rings, forgetting the topology. This is a subject about which relatively little is known, though important contributions have been made by Higson [Hig], Karoubi [K], Suslin [S], Fischer [F] and Prasolov [Pr], and Suslin-Wodzicki [SW]. The topological $K$-theory of operator algebras, on the other hand, is well studied, and is the source of many of the important advances that have been made in operator algebras in recent years. The natural tool in the study of algebraic $K$-theory of $C^{*}$-algebras, or more generally of Banach algebras, is therefore the comparison map (see Theorem 1.1) between these two theories, and the most interesting results say roughly that this map is in some sense close to being an isomorphism. Any result of this type may be viewed as a $K$-theoretic sort of automatic continuity theorem. The most famous such theorem (though also the least interesting) is the one that says that $K_{0}$ is the same, whether computed in the algebraic or the topological category.

One might legitimately ask the purpose of studying purely algebraic invariants of topological objects such as $C^{*}$-algebras, especially since there is no obvious reason to expect them to be well behaved. One might therefore give the following two-fold motivation:

(1) In cases where the comparison map is close to an isomorphism, it shows that operator algebras are quite well behaved from a purely algebraic point of view. For example, we will see in Section 3 that commutative $C^{*}$-algebras are $K$-regular, in other words, behave from the $K$-theoretic point of view like regular rings (Noetherian rings of finite homological dimension).

1991 Mathematics Subject Classification. Primary 19K99; Secondary 19D50, 19D35, 19L41, 47C15.

Key words and phrases. $C^{*}$-algebra, algebraic $K$-theory, $K$-regularity, $K$-theory with finite coefficients, stable rank, Karoubi-Villamayor $K$-groups.

This research was partially supported by NSF grants DMS-90-02642 and DMS-92-25063.

Typeset by $\mathcal{A M}_{\mathcal{M}}-\mathrm{T}_{\mathrm{E}} \mathrm{X}$ 
(2) In cases where the comparison map is not always an isomorphism, it suggests ways to define new invariants of operator algebras, with many of the properties of topological $K$-groups, but with a richer structure. For example, the negative algebraic $K$-theory of commutative $C^{*}$-algebras recaptures connective $K$-theory (Theorem 2.3 below), a more subtle invariant of spaces than periodic $K$-theory. (Periodic $K$-theory can be recovered from connective $K$-theory, but not vice versa.) And algebraic $K$-theory, unlike topological $K$-theory, does not always commute with $C^{*}$-algebraic direct limits (though it commutes with uncompleted direct limits).

In addition, there are some problems in operator algebra theory (such as those related to the lifting of nilpotent elements $[\mathrm{OP}]$ ) that naturally involve algebraic $K$-theory and not topological $K$-theory. The results of this paper might be useful for such problems.

Section 1 of the paper summarizes various facts about algebraic $K$-theory of operator algebras that are already in the literature or known to the experts, though perhaps hard to locate in the form we will need. Section 2 introduces a number of conjectures about negative $K$-theory and $K$-regularity. One of the main results, proving one of these conjectures for commutative $C^{*}$-algebras, takes up Section 3. Section 4 concerns extensions and generalizations of the Fischer-Prasolov Theorem comparing algebraic and topological $K$-theory with finite coefficients. Section 5 contains a few interesting facts about polynomial rings over commutative $C^{*}$-algebras, which were discovered in the course of another attack on the problems studied in Sections 2 and 3. These might serve as prototypes for additional studies of rings of this sort.

Notation. Many of the results of this paper hold for operator algebras over both $\mathbb{R}$ and $\mathbb{C}$. To avoid having to write " $\mathbb{R}$ or $\mathbb{C}$ " over and over again, we use the symbol $\mathbb{F}$ to denote the field of scalars, in situations where the arguments work over both $\mathbb{R}$ and $\mathbb{C}$. The algebra of compact operators on an infinite-dimensional separable Hilbert space over $\mathbb{F}$ is denoted as usual by $\mathcal{K}$. If $A$ is a ring (say with unit), $\mathbb{K}(A)$ will denote its (non-connective) algebraic $K$-theory spectrum, whose homotopy groups are the Quillen $K$-groups in positive degrees and the Bass negative $K$-groups in negative degrees. Various constructions of this spectrum are known, for example those of Gersten [Ger] or of Wagoner [Wag], and all the constructions are known to be naturally equivalent. The algebraic $K$-groups of $A$ with finite coefficients are defined to be the homotopy groups of the smash-product of $\mathbb{K}(A)$ with a Moore space. If $A$ is an $\mathbb{F}$-algebra without unit, $A_{+}$will denote its unitalization $A+\mathbb{F} \cdot 1$. If $A$ is a Banach algebra, $\mathbb{K}^{\text {top }}(A)$ will denote the topological $K$-theory spectrum of $A$, whose homotopy groups are the topological $K$-groups. (This is a non-connective spectrum constructed out of the classifying space $B G L^{\text {top }}(A)$ for the general linear group, viewed as a topological group.) Bott periodicity gives a natural equivalence of this spectrum with its double or 8-fold loop space (in the real and complex cases, respectively).

\section{REVIEW OF KNOWN FACTS}

In this section, we review a number of known facts about algebraic $K$-theory of $C^{*}$ algebras. Some of these are explicitly in the literature and others are only implicitly there but known to the experts. However, even in the latter case, we claim no great originality for anything in this section.

The most fundamental fact of all, which we will use over and over, is the following.

1.1. Theorem. Given a unital Banach algebra $A$ (over $\mathbb{F}=\mathbb{R}$ or $\mathbb{C}$ ), there is a map of spectra $\mathbb{K}(A) \rightarrow \mathbb{K}^{\text {top }}(A)$ which is functorial in $A$, which induces the identity map 
$K_{0}(A) \rightarrow K_{0}^{\mathrm{top}}(A)=K_{0}(A)$, and which induces the map $B G L^{\mathrm{disc}}(A)^{+} \rightarrow B G L^{\mathrm{top}}(A)$ corresponding to the identity map $G L^{\text {disc }}(A) \rightarrow G L^{\text {top }}(A)$. Similarly, if I is a (2-sided) ideal in $A$, there is a functorial map $\mathbb{K}(A, I) \rightarrow \mathbb{K}^{\mathrm{top}}(A, I)$ with similar properties.

Proof. This fact is well known and was stated without proof in [Ro1, §2.1], but we review the argument. Certainly the identity map of topological groups $G L^{\operatorname{disc}}(A) \rightarrow G L^{\mathrm{top}}(A)$ induces a map of classifying spaces $B G L^{\text {disc }}(A) \rightarrow B G L^{\text {top }}(A)$ and then a functorial (infinite loop) map of infinite loop spaces

$$
K_{0}(A) \times B G L^{\mathrm{disc}}(A)^{+} \rightarrow K_{0}(A) \times B G L^{\mathrm{top}}(A) .
$$

It is necessary to "deloop" this map. This can be done inductively, using the identification of the Laurent polynomial ring $A\left[t, t^{-1}\right]$ with a dense subring of $C^{\mathbb{C}}\left(S^{1}\right) \widehat{\otimes} A$ (in the complex case) or with a dense subring of $C\left(S^{-1}\right) \widehat{\otimes} A$ in the real case. Here $\widehat{\otimes}$ denotes "completed tensor product," with respect to the sup norm for functions $S^{1} \rightarrow A_{\mathbb{C}}$, and the notation $C\left(S^{-1}\right)$ comes from $K R$-theory, and refers to the algebra of functions in $C^{\mathbb{C}}\left(S^{1}\right)$ satisfying $f(\bar{z})=\overline{f(z)}$. (Identify $t$ with the function $z$ on the unit circle $S^{1}$ in the complex plane, and use Stone-Weierstrass.) By Bott periodicity for $K U$ and $K R, \mathbb{K}^{\text {top }}\left(C^{\mathbb{C}}\left(S^{1}\right) \widehat{\otimes} A\right)$ and $\mathbb{K}^{\text {top }}\left(C\left(S^{-1}\right) \widehat{\otimes} A\right)$ each split as $\mathbb{K}^{\text {top }}(A) \oplus \Sigma \mathbb{K}^{\text {top }}(A)$, and the splitting is compatible with the Bass-Heller-Swan splitting of $\mathbb{K}\left(A\left[t, t^{-1}\right]\right)$ as $\mathbb{K}(A) \oplus \Sigma \mathbb{K}(A) \oplus$ Nil-terms. So (writing things out only for the complex case, for simplicity) consider the diagram

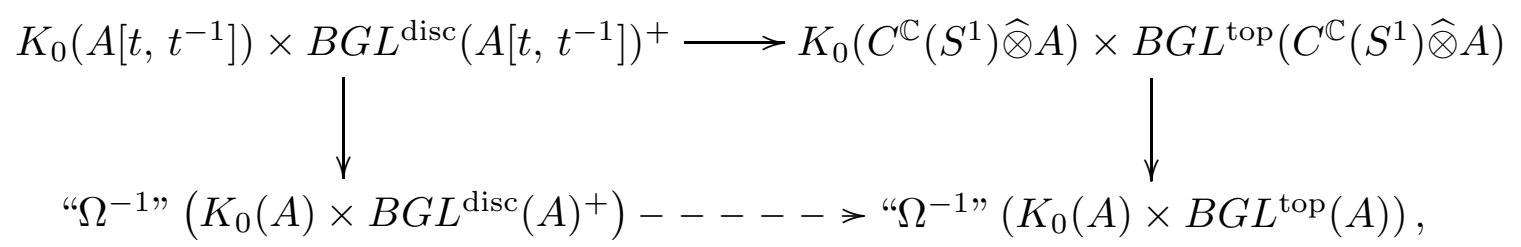

where the vertical maps project onto the appropriate summand. The induced dashed arrow at the bottom makes it possible to deloop once. Then continue by induction. The relative case is done similarly.

1.2. Theorem [SW, Theorem 10.1 and Corollary 10.4]. Any Banach algebra A (over $\mathbb{F}=\mathbb{R}$ or $\mathbb{C}$ ) with left or right bounded approximate unit, and in particular any $C^{*}$-algebra, is $H$-unital and satisfies excision in algebraic $K$-theory. (In particular, if $A$ is non-unital and is embedded as an ideal in a larger Banach algebra $B$, the relative $K$-theory spectrum $\mathbb{K}(B, A)$ is naturally equivalent to $\mathbb{K}\left(A_{+}, A\right)$, regardless of the choice of $B$, hence it makes sense to call it the $K$-theory spectrum $\mathbb{K}(A)$ of $A$.)

1.3. Theorem $[\mathrm{SW}]$. Any Banach algebra $A$ (over $\mathbb{F}=\mathbb{R}$ or $\mathbb{C}$ ) with bounded approximate unit, and in particular any $C^{*}$-algebra, satisfies excision for $N^{r} K$-theory for any $r \geq 1$.

Proof. This follows from Theorem 1.2, from another application of the main theorem of [SW], and from [SW, Theorem 7.10], which implies that any polynomial ring over an $H$-unital algebra is again $H$-unital.

1.4. Theorem. Let $A$ be a stable $C^{*}$-algebra (over $\mathbb{F}=\mathbb{R}$ or $\mathbb{C}$ ) (that is, a $C^{*}$-algebra for which $A \cong A \widehat{\otimes} \mathcal{K})$. Then the map $\mathbb{K}(A) \rightarrow \mathbb{K}^{\text {top }}(A)$ of Theorem 1.1 is an equivalence.

Proof. In [SW], this is listed as Theorem 10.9, but only for the complex case and for the connective $K$-theory spectra. However, the dimension-shifting argument in the proof of 
[Hig, Theorem 5.3.3], which showed that if $A$ is a stable $\sigma$-unital $C^{*}$-algebra with multiplier algebra $\mathcal{M}(A)$, then

$$
K_{i}(\mathcal{M}(A), A) \rightarrow K_{i}^{\mathrm{top}}(\mathcal{M}(A), A)
$$

is an isomorphism for $i \geq 0$, works equally well the other way to give similar isomorphisms for $i<0$. (The stability plays the essential role of guaranteeing Bott periodicity for the algebraic $K$-theory groups; see $[\mathrm{K}]$ for an "explanation.") Hence under these hypotheses, $\mathbb{K}(\mathcal{M}(A), A) \rightarrow \mathbb{K}^{\text {top }}(\mathcal{M}(A), A)$ is an equivalence of spectra. Because of the excision property of Theorem 1.2, it is enough to prove the Theorem for algebras of the form $A \widehat{\otimes} \mathcal{K}$ with $A$ unital, and such stable algebras are $\sigma$-unital. So the result for the complex case follows from Higson's work and from Theorem 1.2. The real case requires redoing the arguments of [Hig], but there is no essential change. Indeed, Higson's key tool, the fact that a stable, split exact functor is homotopy invariant, works in the real case also, because it is based on the foundations of Kasparov's $K K$-theory, which are also available in the real case.

1.5. Theorem. Any stable $C^{*}$-algebra $A$ (over either $\mathbb{R}$ or $\mathbb{C}$ ) is $K_{i}$-regular for all $i$.

Proof. This is proved in [Hig, Lemma 6.3.12] for $i=1$, but exactly the same proof works for all $i$, once one adds Theorem 1.2 (which was not available at the time [Hig] was written).

The following purely operator-theoretic result will also be of interest. For reasons to be explained later, it gave the original motivation for the results of $\S 2$ and $\S 3$ below.

1.6. Theorem [OP, Theorem 6.7]. Let $A$ be any $C^{*}$-algebra (over $\mathbb{C}$ ) and let $I$ be a closed ideal in $A$. Then any element $y \in A / I$ satisfying $y^{n}=0$ can be lifted to an element $x \in A$ with $x^{n}=0$.

Remark. The same statement is also true for real $C^{*}$-algebras, as one can see using very slight modifications in the argument.

In general, higher algebraic $K$-theory of $C^{*}$-algebras differs considerably from topological $K$-theory. However, there is a remarkable result using $K$-theory with finite coefficients.

1.7. Theorem ([S] in the case of $\mathbb{R}$ and $\mathbb{C} ;[\mathrm{F}]$ and $[\mathrm{P}]$ in general). Let $A$ be a commutative $C^{*}$-algebra. Then the map of Theorem 1.1 induces isomorphisms of $K$-groups with finite coefficients

$$
K_{i}(A ; \mathbb{Z} / k) \rightarrow K_{i}^{\mathrm{top}}(A ; \mathbb{Z} / k), \quad i \geq 1
$$

for any $k>1$.

Remark on the proof. The Theorem for the cases $A=C^{\mathbb{R}}(X)$ and $A=C^{\mathbb{C}}(X)$ (X compact Hausdorff) is proved in $[\mathrm{F}]$ and $[\mathrm{P}]$. Then by excision (Theorem 1.2), the Theorem also holds for the non-unital cases $A=C_{0}^{\mathbb{R}}(X)$ and $A=C_{0}^{\mathbb{C}}(X)$ (X locally compact Hausdorff). (This case of the theorem is already in [F, Proposition 8.1].) The most general commutative complex $C^{*}$-algebra is of the form $C_{0}^{\mathbb{C}}(X)$, so we're done with the complex case.

Now the most general real $C^{*}$-algebra is

$$
C_{0}(X, \tau) \underset{\mathrm{def}}{=}\left\{f \in C_{0}^{\mathbb{C}}(X) \mid f(\tau(x))=\overline{f(x)}\right\},
$$

where $(X, \tau)$ is a Real space in the sense of Atiyah, i.e., a locally compact space equipped with an self-homeomorphism $\tau$ such that $\tau^{2}=1$. The topological $K$-groups of $C_{0}(X, \tau)$ are 
Atiyah's $K R$-groups of $(X, \tau)$ (with compact supports). We have a short exact sequence of real $C^{*}$-algebras

$$
0 \rightarrow C_{0}\left(X \backslash X^{\tau}, \tau\right) \rightarrow C_{0}(X, \tau) \rightarrow C_{0}^{\mathbb{R}}\left(X^{\tau}\right) \rightarrow 0
$$

which gives a commutative diagram of fibrations of $K$-theory spectra

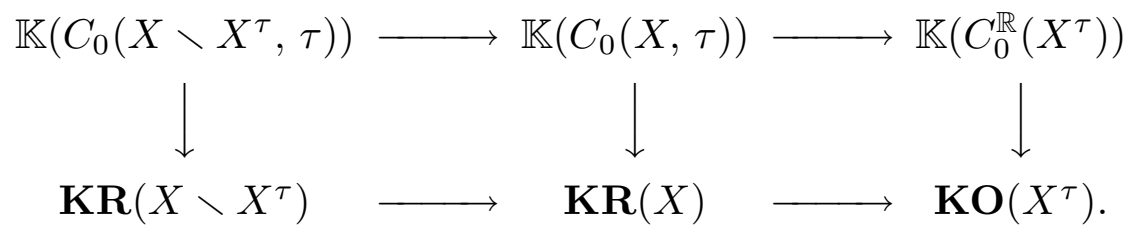

Applying the Five-Lemma to the corresponding long exact sequences of $K$-groups gives the result for $C_{0}(X, \tau)$ once we know it for $C_{0}\left(X \backslash X^{\tau}, \tau\right)$ and for $C_{0}^{\mathbb{R}}\left(X^{\tau}\right)$.

So, replacing $X$ by $X \backslash X^{\tau}$, we may (and do) assume $\tau$ acts freely. Then if the covering map $q: X \rightarrow X / \tau$ is a trivial covering, i.e., if $X \cong Y \amalg Y$, with $\tau$ interchanging the two copies of $Y$, we have $C_{0}(X, \tau) \cong C_{0}^{\mathbb{C}}(Y)$, and the Theorem follows from the complex case. By Mayer-Vietoris, we get the result whenever $\tau$ acts freely on $X$ and there is a finite cover of $X / \tau$ by open sets over which the covering $X \rightarrow X / \tau$ is trivial, for example when $X$ is compact. To handle the general locally compact case, one can first argue as in $[\mathrm{F}$, $\S 8]$ that the inclusion $C_{c}(X, \tau) \hookrightarrow C_{0}(X, \tau)$ induces isomorphisms on algebraic $K$-groups with finite coefficients. Since $X / \tau$ is the increasing limit of open subsets $U_{j}$ with compact closures over which $q$ is trivial, $C_{c}(X, \tau)$ is the inductive limit of (non-unital) rings of functions $C_{c}\left(q^{-1}\left(U_{j}\right), \tau\right)$ for which the theorem is already verified. So

$$
\begin{aligned}
K_{i}\left(C_{0}(X, \tau) ; \mathbb{Z} / k\right) \cong K_{i}\left(C_{c}(X, \tau) ; \mathbb{Z} / k\right) \cong \underline{\lim _{\longrightarrow}} K_{i}\left(C_{c}\left(q^{-1}\left(U_{j}\right), \tau\right) ; \mathbb{Z} / k\right) \\
\cong \underline{\lim } K R^{-i}\left(q^{-1}\left(U_{j}\right), \tau ; \mathbb{Z} / k\right)=K R^{-i}(X ; \mathbb{Z} / k)
\end{aligned}
$$

The correct spectrum-theoretic version of this is essentially proved in [Ro1, Theorem $2.2]$, and can be reformulated as follows. For simplicity we only give the results for $C_{0}^{\mathbb{R}}(X)$ and $C_{0}^{\mathbb{C}}(X)$, though there is a similar result for $C_{0}(X, \tau)$ using $\mathbf{k r}$.

1.8. Theorem. The map of Theorem 1.1 with finite coefficients

$$
\mathbb{K}(A ; \mathbb{Z} / k) \rightarrow \mathbb{K}^{\text {top }}(A ; \mathbb{Z} / k),
$$

for $A=C_{0}^{\mathbb{R}}(X)$ or $C_{0}^{\mathbb{C}}(X)$, induces (for $X$ a locally compact space whose one-point compactification $X_{+}$has the homotopy type of a finite $C W$-complex) equivalences of spectra

$$
\mathbb{K}\left(C_{0}^{\mathbb{R}}(X) ; \mathbb{Z} / k\right) \rightarrow \mathbf{k o}(\mathbb{Z} / k) \wedge X_{+}, \quad \mathbb{K}\left(C_{0}^{\mathbb{C}}(X) ; \mathbb{Z} / k\right) \rightarrow \mathbf{k u}(\mathbb{Z} / k) \wedge X_{+},
$$

where $\mathbf{k o}(\mathbb{Z} / k)$ and $\mathbf{k u}(\mathbb{Z} / k)$ denote the spectra of connective $K$-theory with $\mathbb{Z} / k$ coefficients.

Proof. The real and complex cases are exactly the same, so we only give the proof in the real case. Let $H^{-i}(X)=K_{i}\left(C_{0}^{\mathbb{R}}(X) ; \mathbb{Z} / k\right)$. By Theorem 1.7 , this is a homotopy functor of $X$ for $i>0$, and as proved in [Ro1, §2], it is also a homotopy functor for $i \leq 0$. Excision and the long exact sequence for $K$-theory with finite coefficients then show that $H$ is a(n 
unreduced) cohomology theory (on the category of unbased locally compact spaces and proper maps), so that when $X_{+}$has the homotopy type of a finite CW-complex,

$$
\mathbb{K}\left(C_{0}^{\mathbb{R}}(X) ; \mathbb{Z} / k\right) \simeq \mathbb{E} \wedge X_{+}
$$

for some spectrum $\mathbb{E}$. It remains to identify $\mathbb{E}$. The map

$$
\mathbb{K}\left(C_{0}^{\mathbb{R}}(X) ; \mathbb{Z} / k\right) \rightarrow \mathbb{K}^{\text {top }}\left(C_{0}^{\mathbb{R}}(X) ; \mathbb{Z} / k\right)
$$

induces a natural transformation of cohomology theories from $H$ to $K O(\ldots \mathbb{Z} / k)$ which is an isomorphism on $H^{-i}$ for $i \geq 0$ by Theorem 1.7 , and furthermore, $H^{i}(p t)=K_{-i}(\mathbb{R} ; \mathbb{Z} / k)$ $=0$ for $i>0$. So the cohomology theory $H$ is connective. By the universal property of the connective cover of a spectrum [Ad, p. 145], we have a factorization

$$
H \rightarrow k o(\ldots \mathbb{Z} / k) \rightarrow K O(\ldots ; \mathbb{Z} / k) \text {. }
$$

Since the natural transformation $H^{-i}(X) \rightarrow{\widetilde{k_{O}}}^{-i}\left(X_{+} ; \mathbb{Z} / k\right)$ is an isomorphism for $X$ a point, it is an isomorphism for any $X$ for which $X_{+}$has the homotopy type of a finite CW-complex.

\section{2. $K$-REgUlarity AND HOMOTOPY INVARIANCE OF NEGATIVE ALGEBRAIC $K$-THEORY}

Let us now discuss some of the evidence for the following:

2.1. Conjecture. Any $C^{*}$-algebra (over $\mathbb{F}=\mathbb{R}$ or $\mathbb{C}$ ) is $K_{0}$-regular.

For abelian $C^{*}$-algebras of the form $C_{0}^{\mathbb{R}}(X)$ or $C_{0}^{\mathbb{C}}(X)$, this was proved in [Ro1, Theorem 2.5]. Another proof of a much stronger result will be given in the next section. For stable $C^{*}$-algebras, Conjecture 2.1 is a special case of Theorem 1.5. And a result along these lines is strongly suggested by the Olsen-Pedersen Theorem, 1.6. Indeed, if $A$ is a $C^{*}$-algebra and $I$ is a closed ideal in $A$, then the short exact sequence

$$
0 \rightarrow I \rightarrow A \rightarrow A / I \rightarrow 0
$$

gives rise by Theorem 1.3 to a long exact sequence of $N K$-groups

$$
\ldots \stackrel{\partial}{\rightarrow} N K_{i}(I) \rightarrow N K_{i}(A) \rightarrow N K_{i}(A / I) \stackrel{\partial}{\rightarrow} N K_{i-1}(I) \rightarrow \ldots
$$

(Actually, we only need the part of the sequence from $N K_{1}(A)$ to $N K_{0}(I)$, which is well-defined even without appealing to Suslin-Wodzicki, since $N K_{0}$ always has the excision property.) Since $N K_{1}$ is naturally isomorphic to the functor Nil constructed out of nilpotent matrices, and since a matrix algebra over a $C^{*}$-algebra is again a $C^{*}$-algebra, the Olsen-Pedersen Theorem, 1.6, applied to $M_{k}(A)$ for all $k \in \mathbb{N}$, implies that the map $N K_{1}(A) \rightarrow N K_{1}(A / I)$ is always surjective, and thus that $\partial: N K_{1}(A / I) \rightarrow N K_{0}(I)$ is always the 0-map. Vanishing of $N K_{0}(I)$ would of course "explain" this phenomenon.

In fact, we can turn things around and use Theorem 1.6 to give a proof, not depending on the harder results in [Hig], of the vanishing of $N K_{0}$ for stable $C^{*}$-algebras. Let $A$ be a stable $C^{*}$-algebra and let $\mathcal{M}(A)$ be its multiplier algebra. The fact that this algebra is highly "infinite" implies that all its $K$-groups, including the $N K$-groups, must vanish [Hig, Theorem 2.6.5]. So from the exact sequence

$$
N K_{1}(\mathcal{M}(A)) \rightarrow N K_{1}(\mathcal{M}(A) / A) \stackrel{\partial}{\rightarrow} N K_{0}(A) \rightarrow N K_{0}(\mathcal{M}(A)),
$$

we see $\partial: N K_{1}(\mathcal{M}(A) / A) \rightarrow N K_{0}(A)$ is an isomorphism. But as we just saw, the OlsenPedersen Theorem implies that $\partial$ is the 0 -map, so $N K_{0}(A)=0$.

Similarly, the results of $\S 1$ also provide evidence for: 
2.2. Conjecture. The negative $K$-functors $K_{i}, i \leq 0$, are homotopy functors on the category of $C^{*}$-algebras (over $\mathbb{F}=\mathbb{R}$ or $\mathbb{C}$ ).

Remark. Homotopy invariance means that if $B$ and $A$ are $C^{*}$-algebras and

$$
\varphi: B \rightarrow C([0,1]) \widehat{\otimes} A
$$

is a homotopy between homomorphisms

$$
\varphi_{0}=\operatorname{eval}_{0} \circ \varphi, \quad \varphi_{1}=\operatorname{eval}_{1} \circ \varphi: B \rightarrow A
$$

(here $e v a l_{0}, e v a l_{1}$ are the evaluation maps $C([0,1]) \widehat{\otimes} A \rightarrow A$ at 0 and 1 ), then the induced maps on negative $K$-groups coincide. To prove this, it is clearly sufficient to forget the algebra $B$ and to show that the evaluation maps

$$
\text { eval }_{0} \text { and } \text { eval }_{1}: C([0,1]) \widehat{\otimes} A \rightarrow A
$$

induce the same maps on negative $K$-groups. For this it is enough to prove vanishing of the negative $K$-groups for the "cone" on $A, c A=C_{0}((0,1]) \widehat{\otimes} A$, since we have exact sequences of $C^{*}$-algebras

$$
0 \rightarrow C_{0}((0,1]) \widehat{\otimes} A \rightarrow C([0,1]) \widehat{\otimes} A \stackrel{\text { eval }_{j}}{\longrightarrow} A \rightarrow 0
$$

for $j=0,1$, each of which is split by the inclusion of $A$ into $C([0,1]) \widehat{\otimes} A$ as constant functions, and we can apply the associated long exact $K$-theory sequences.

The abelian case of Conjecture 2.2, proved in [Ro1], leads to Theorem 2.4 of [Ro1]. For completeness, we also state a version for $K R$-theory.

2.3. Theorem [Ro1, Theorem 2.4]. Let $X$ be a (second-countable) locally compact space. Then there are natural isomorphisms

$$
\begin{array}{ll}
K_{i}\left(C_{0}^{\mathbb{C}}(X)\right) \cong \widetilde{k u}^{-i}\left(X_{+}\right), & i \leq 0, \\
K_{i}\left(C_{0}^{\mathbb{R}}(X)\right) \cong \widetilde{k o}^{-i}\left(X_{+}\right), & i \leq 0,
\end{array}
$$

and for a Real space $(X, \tau)$ in the sense of Atiyah

$$
K_{i}\left(C_{0}(X, \tau)\right) \cong \widetilde{k r}^{-i}\left(X_{+}\right), \quad i \leq 0 .
$$

Here $X_{+}$denotes the one-point compactification of $X$ and $k u$, ko, and $k r$ denote the connective $K$-theories.

Proof. Homotopy-invariance of the maps $X \mapsto K_{i}\left(C_{0}^{\mathbb{C}}(X)\right)$ and $X \mapsto K_{i}\left(C_{0}^{\mathbb{R}}(X)\right)(i \leq$ $0)$, together with the long exact sequences in $K$-theory, shows that these are countably additive cohomology theories on the category of (second-countable) locally compact spaces and proper maps. These can be spliced together with $K$-theory with compact supports (for $i \geq 0$ ) to get cohomology theories that must be representable by spectra. These spectra are connective, since negative $K$-theory vanishes for fields, and thus $K_{i}\left(C_{0}^{\mathbb{C}}\left(S^{0}\right)\right)$ and $K_{i}\left(C_{0}^{\mathbb{R}}\left(S^{0}\right)\right)$ vanish for $i \leq 0$. Since we have natural transformations to topological 
$K$-theory coming from the map of Theorem 1.1, which must factor through connective $K$ theory, and since the induced maps to connective $K$-theory give isomorphisms of coefficient groups, they are isomorphisms of cohomology theories. This proves the theorem in the real and complex cases.

The Real case is handled as in the proof of Theorem 1.7.

2.4. Remark. Here are some possible strategies for attacks on Conjectures 2.1 and 2.2. Unfortunately, neither seems to work without some additional information. We illustrate these strategies in the case of Conjecture 2.1. Similar methods could be applied to Conjecture 2.2.

Strategy A: Stabilization. One idea is to exploit the fact that we understand the $K$-groups and $N K$-groups of stable $C^{*}$-algebras. Suppose $A$ is a $C^{*}$-algebra and we are given a class in $K_{0}\left(A\left[t_{1}, \ldots, t_{r}\right]\right)$ which maps to 0 in $K_{0}(A)$ under the map $q_{*}$ induced by $q: t_{j} \rightarrow 0$. We may represent the class by a formal difference $[e]-\left[e^{\prime}\right]$ of idempotent matrices over $A\left[t_{1}, \ldots, t_{r}\right]$ such that $q(e)=q\left(e^{\prime}\right)$. Let $\mathcal{H}_{A}$ be the standard Hilbert $A$-module. Its algebra of compact $A$-endomorphisms is $\mathcal{K}\left(\mathcal{H}_{A}\right) \cong A \widehat{\otimes} \mathcal{K}$, which contains as an ideal the algebra $M(A)$ of finite-rank $A$-endomorphisms. (These are in some sense "infinite matrices" over $A$, which explains our choice of notation.) Since $M(A)$ is the algebraic inductive limit of the $A$-endomorphism rings of the finite-rank free $A$-submodules of $\mathcal{H}_{A}$, each of which is isomorphic to a matrix ring over $A, M(A)$ has precisely the same (algebraic) $K$-groups as $A$, and $e, e^{\prime} \in M(A)\left[t_{1}, \ldots, t_{r}\right]$. Let $\iota: M(A) \rightarrow \mathcal{K}\left(\mathcal{H}_{A}\right) \cong A \widehat{\otimes} \mathcal{K}$ be the completion map. Then $\iota_{*}\left([e]-\left[e^{\prime}\right]\right) \in N^{r} K_{0}(A \widehat{\otimes} \mathcal{K})=0$ (Theorem 1.5). Thus the projective $\mathcal{K}\left(\mathcal{H}_{A}\right)\left[t_{1}, \ldots, t_{r}\right]_{+}$-modules generated by $e$ and $e^{\prime}$ are stably isomorphic. Suppose we could show they were actually isomorphic (using stable-range techniques to cancel the additional summands needed for stabilization), by an isomorphism sending $e$ to $e^{\prime}$. Then the isomorphism of $\mathcal{K}\left(\mathcal{H}_{A}\right)\left[t_{1}, \ldots, t_{r}\right]_{+}$-modules would restrict to an isomorphism of $M(A)\left[t_{1}, \ldots, t_{r}\right]_{+}$-modules.

This strategy would work immediately if one could show that $C=A \widehat{\otimes} \mathcal{K}\left[t_{1}, \ldots, t_{r}\right]$ has Bass stable rank one, since in that case one has cancellation for arbitrary projective modules [Va1, Theorem 2.7]. Because of the tensor factor $\mathcal{K}$, the stable rank of $C$, if finite, is at most two; however, stable rank one seems unlikely since it fails already when $A=\mathbb{C}$. (Stable rank one for $\mathcal{K}\left[t_{1}, \ldots, t_{r}\right]$ would imply stable rank one for $\mathbb{C}\left[t_{1}, \ldots, t_{r}\right]$ by [Va1, Theorem 3.9], which simply isn't true; for $r \geq 1$, the polynomial ring always has stable rank at least 2.)

Strategy B: Topologization. Another possible idea, used with some success in [Ro1], is to topologize the algebra $B=A\left[t_{1}, \ldots, t_{r}\right]$, and then the matrix algebras over this algebra, with the inductive limit topology for the subsets $B_{d}$ of polynomials of fixed maximal degree $d$. (The polynomials over $A$ of degree $\leq d$ in the $t_{j}$ 's are a finitely generated free $A$-module, and thus carry a natural norm topology. Obviously $B$ is the countable increasing union $\bigcup_{d=0}^{\infty} B_{d}$.) The inductive limit topology is compactly generated and Hausdorff, and is defined by the property that a map from a compact Hausdorff space $X$ into $B$ is continuous if and only if it has image contained in a single $B_{d}$, and is continuous as a map $X \rightarrow B_{d}$.

Without loss of generality, assume that $A$ has a unit. Fix an $n$ and consider the space of idempotents $P_{n}(B)$ in $M_{n}(B)$; this has an obvious inductive limit topology as the union of the $P_{n}\left(B_{d}\right)$ 's (with obvious notation). Define an equivalence relation $\sim$ on $P_{n}(B)$ by $e \sim e^{\prime}$ if $B^{n} e \cong B^{n} e^{\prime}$ as $B$-modules. By definition of $K_{0}, P_{n}(B) / \sim$ maps naturally to $K_{0}(B)$, 
and $K_{0}(B)$ is the Grothendieck group of the monoid $\lim _{n} P_{n}(B) / \sim$. We give $P_{n}(B) / \sim$ the quotient topology from $P_{n}(B)$. Then we have the following result.

2.5. Proposition. With notation as above, suppose $P_{n}(B) / \sim$ is Hausdorff and discrete for all sufficiently large $n$. Then $N^{j} K_{0}(A)=0$ for $j \leq r$.

Proof. For $s \in[0,1]$, let $\varphi_{s}$ be the endomorphism of $B$ defined by $t_{j} \mapsto s t_{j}$. Then $\varphi_{1}=i d_{B}$ and $\varphi_{0}=q: B \rightarrow A$. Furthermore, each $\varphi_{s}$ induces a self-map of $P_{n}(B) / \sim$, and the map

$$
\varphi_{*}:\left(P_{n}(B) / \sim\right) \times[0,1] \rightarrow P_{n}(B) / \sim
$$

is continuous. (The map $P_{n}(B) \times[0,1] \rightarrow P_{n}(B)$ is clearly continuous for the inductive limit topology, since $\varphi_{s}$ does not increase the degree of any polynomial, and then passes to the quotient.) Thus in $P_{n}(B) / \sim, \varphi_{1}(e)=e$ and $\varphi_{0}(e)=q(e)$ lie in the same equivalence class. If this is true for all sufficiently large $n$, then $q_{*}$ is an isomorphism on $K_{0}$.

The hypothesis of Proposition 2.5 is true if $A=\mathbb{C}$ or $\mathbb{R}$, but unfortunately it seems hard to determine when it holds in general. In fact, it seems as if usually it would amount to putting the cart before the horse - usually it would be easier to prove vanishing of $N K_{0}$ than the hypothesis of the Proposition, even for $d=1$. However, the same method appears more useful when applied to Conjecture 2.2. We have similarly:

2.6. Proposition. Let $B$ be the Laurent polynomial ring $B=A\left[t_{1}^{ \pm}, \ldots, t_{r}^{ \pm}\right]$, and define an inductive limit topology as before on $P_{n}(B)$ as before, but replacing the degree of a monomial $t_{1}^{i_{1}} \ldots t_{r}^{i_{r}}$ by its "length" $\left|i_{1}\right|+\cdots+\left|i_{r}\right|$. Again, assume $P_{n}(B) / \sim$ is Hausdorff and discrete for all sufficiently large $n$. Then $K_{-j}(A)$ is homotopy-invariant for $j \leq r$, in the sense that homotopic maps into $A$ induce the same map on $K_{-j}(A)$.

Proof. An idempotent matrix $e$ in $M_{n}\left(C([0,1], A)\left[t_{1}^{ \pm}, \ldots, t_{r}^{ \pm}\right]\right)$is by definition of the inductive limit topology the same thing as a continuous map $[0,1] \rightarrow P_{n}(B)$. As before, discreteness of $P_{n}(B) / \sim$ implies this map passes to a constant map $[0,1] \rightarrow P_{n}(B) / \sim$. If $e$ and $e^{\prime}$ are two maps $[0,1] \rightarrow P_{n}(B)$ corresponding to projective modules that become equivalent after evaluating at 0 , they are thus equivalent after evaluating anywhere, so the maps $e v a l_{0}$ and $e v a l_{1}$ agree. If this is true for all large $n$, then $K_{0}(B)$ is homotopy-invariant with respect to homomorphisms from other Banach algebras into $A$. By the argument of Proposition 2.5, this forces $N^{j} K_{0}(A)$ to vanish for $j \leq r$, and $K_{-j}(A)$ for $j \leq r$, which is a canonical summand in $K_{0}(B)$, is homotopy-invariant.

It is natural to ask whether Conjectures 2.1 and 2.2 should apply more generally to Banach algebras, or whether they are special to the case of $C^{*}$-algebras. The following counterexamples show that the latter is the case.

2.7. Example: A (commutative, semisimple) Banach algebra which is not $K_{0^{-}}$ regular. Let $A$ be the disk algebra (the algebra of continuous complex-valued functions on the closed unit disk $\bar{\Delta}$ in the complex plane which are holomorphic in the open disk $\Delta$ ), and let $I$ be the ideal in $A$ generated by the function $z^{2}$, in other words, the functions in $A$ which vanish to second order at 0 . Clearly $A / I \cong \mathbb{C}[z] /\left(z^{2}\right)$. The element $z \in A / I$ is nilpotent, and thus corresponds to an element [z] of $\mathrm{Nil} A / I \cong N K_{1}(A / I)$. We claim it does not lift to an element of $\operatorname{Nil} A \cong N K_{1}(A)$, and that $\partial[z] \neq 0$ in $N K_{0}(I)$. To see this, 
note that since $A[t]$ is commutative, we have an exact sequence (obtained from the long exact $K$-theory sequence by dividing out $S K_{1}$-terms)

$$
\cdots \rightarrow A[t]^{\times} \rightarrow(A / I)[t]^{\times} \stackrel{\partial}{\rightarrow} K_{0}(I[t]) .
$$

The element $[z] \in \operatorname{Nil} A / I$ corresponds to the element

$$
1+z t \in(A / I)[t]^{\times}=\left(\mathbb{C}[z, t] /\left(z^{2}\right)\right)^{\times}
$$

Since $A$ is an integral domain, an invertible element of $A[t]$ cannot contain any non-zero terms divisible by $t$. (If $n>0, m \geq 0, a_{n} \neq 0, b_{m} \neq 0$, and

$$
\left(a_{0}+\cdots+a_{n} t^{n}\right)\left(b_{0}+\cdots+b_{m} t^{m}\right)=1,
$$

then $a_{n} b_{m}=0$, a contradiction.) So $1+z t$ does not lift to an invertible element of $A[t]$, and must map non-trivially to the $N K_{0}(I)$ summand in $K_{0}(I[t])$. Note that $I_{+}$is thus a unital semisimple Banach algebra which is not $K_{0}$-regular.

2.8. Example: Failure of homotopy invariance of $N K_{0}$ for Banach algebras. The same example shows as well that the functor $N K_{0}$ is not homotopy-invariant on general Banach algebras, even if they are semisimple. Indeed, define a homotopy $\varphi: A \times[0,1] \rightarrow A$ from the identity map $\varphi_{1}=i d_{A}$ to $\varphi_{0}=\operatorname{eval}_{0}$ by $\left(\varphi_{t} f\right)(z)=f(t z),|z| \leq 1,0 \leq t \leq 1$. Then each $\varphi_{t}$ preserves the ideal $I$ and so $\varphi$ restricts to a homotopy from the identity map on $I$ to the 0 map; i.e., $I$ is contractible. However, we have seen that $N K_{0}(I) \neq 0$. If $N K_{0}$ were a homotopy functor, this would be impossible.

\section{3. $K$-REGULARITY RESULTS}

In this section we will show that abelian $C^{*}$-algebras, in spite of being highly nonNoetherian, and thus somewhat pathological from the algebraist's usual point of view, are in fact quite well behaved when it comes to algebraic $K$-theory. In particular, their Karoubi-Villamayor $K$-groups coincide with their usual algebraic $K$-groups.

3.1. Theorem. Any commutative $C^{*}$-algebra $A$ (over $\mathbb{F}=\mathbb{R}$ or $\mathbb{C}$ ) is $K_{i}$-regular for all $i$, i.e., the inclusion $A \hookrightarrow A\left[t_{1}, \ldots, t_{r}\right]$ induces isomorphisms on all algebraic $K$-groups, for any $r$.

As the proof is rather complicated, let us begin with an easier result which is somewhat related and which may give the reader some idea why this should be true. In the case of commutative algebras, $K_{0}$-regularity is closely linked to the subject of Pic-regularity. In this regard, it is convenient to note the following.

3.2. Theorem. Any commutative $C^{*}$-algebra $A$ (over $\mathbb{F}=\mathbb{R}$ or $\mathbb{C}$ ) is Pic-regular, i.e., the inclusion $A \hookrightarrow A\left[t_{1}, \ldots, t_{r}\right]$ induces isomorphisms on Pic for all $r \geq 1$.

Proof. We will apply the results of [Swa], which give a criterion for Pic-regularity for general (not necessarily Noetherian) commutative rings. Swan [Swa, Theorem 1] shows that a commutative ring $A$ is Pic-regular if and only if $A_{\text {red }}$ is seminormal, i.e., if $b, c \in A$ and $b^{3}=c^{2}$ implies that there exists some element $a \in A_{\text {red }}$ with $a^{2}=b, a^{3}=c$. For our purposes, since abelian $C^{*}$-algebras are reduced (have no non-zero nilpotent elements), we 
may replace $A_{\text {red }}$ by $A$ here. So suppose $A$ is a commutative $C^{*}$-algebra and $b, c \in A$, $b^{3}=c^{2}$. Realize $A$ as $C_{0}(X, \tau)$ for some locally compact space $X$ and involution $\tau$. We need to construct $a \in A$ with $a^{2}=b, a^{3}=c$. Clearly $b$ and $c$ both vanish on the same closed $\tau$-invariant subset $Y$ of $X$, and $a$ must vanish there as well. So the problem is to construct a suitable square root $a$ of $b$ over the set $W=X \backslash Y$. Replacing $A$ by $C_{0}(W, \tau)$, we may assume $b$ and $c$ are everywhere non-vanishing. Then if $a(x)=\frac{c(x)}{b(x)}$, this is the unique function satisfying $a(x)^{2}=b(x), a(x)^{3}=c(x)$, and is necessarily $\tau$-equivariant and vanishing at infinity (on $W$ ), so it defines an element of $A$. Hence $A$ is seminormal.

Now it is clear that Example 2.7 is closely related to:

3.3. Example: A (semisimple commutative) Banach algebra which is not Picregular. The unital semisimple Banach algebra $I_{+}$of Example 2.7 is not seminormal, hence not Pic-regular. This "explains" why it is not $K_{0}$-regular either. Indeed, this algebra is reduced, and if we choose $b=z^{2}, c=z^{3}$, then $b^{3}=c^{2}$, but $b$ has no square root in $I_{+}$.

3.4. Example: A Special Case of Theorem 3.1. Since the functor $N K_{1}$ is naturally equivalent to the functor Nil constructed from nilpotent matrices, and since $C^{*}$-algebras usually contain many nilpotent elements which cannot be "put in triangular form," one might at first expect $K_{1}$-regularity to fail for general $C^{*}$-algebras, in fact even in the abelian case. To make this more precise, note that if $A=C([0,1])$ (a standard example of a commutative $C^{*}$-algebra), then a class in $N K_{1}(A) \cong$ Nil $A$ is represented by a nilpotent matrix over $A$, which is just a continuous function from $[0,1]$ to nilpotent matrices over $\mathbb{C}$. Consider for example the case where the matrices are of size $2 \times 2$. If this nilpotent matrix-valued function is conjugate under

$$
G L\left(2, C^{\mathbb{C}}([0,1])\right)=C([0,1], G L(2, \mathbb{C}))
$$

to something of the form $\left(\begin{array}{ll}0 & * \\ 0 & 0\end{array}\right)$, it clearly represents the trivial element of $\mathrm{Nil} A$. But there are nilpotent matrix-valued functions which cannot be put in triangular form. Choose the function so that it has rank 1 almost everywhere, but so that the subspaces of $\mathbb{C}^{2}$ representing the kernel and image of the matrix "oscillate" faster and faster as one approaches a point where the matrix degenerates to 0 . There are huge numbers of choices for a matrix function of this desired form, but one possibility is the continuous matrix-valued function

$$
a(x)=\left\{\begin{array}{cc}
x\left(\begin{array}{cc}
\sin \left(\frac{1}{x}\right) \cos \left(\frac{1}{x}\right) & \cos ^{2}\left(\frac{1}{x}\right) \\
-\sin ^{2}\left(\frac{1}{x}\right) & -\sin \left(\frac{1}{x}\right) \cos \left(\frac{1}{x}\right)
\end{array}\right), & x \in(0,1], \\
\left(\begin{array}{ll}
0 & 0 \\
0 & 0
\end{array}\right), & x=0 .
\end{array}\right.
$$

(The continuity follows from the fact that $\sin \left(\frac{1}{x}\right)$ and $\cos \left(\frac{1}{x}\right)$ remain bounded as $x \rightarrow 0_{+}$.) We claim that $a(x)$ is not conjugate under $C([0,1], G L(2, \mathbb{C}))$ to an upper-triangular matrix. For otherwise, there would have to be a continuous map $F$ from $[0,1]$ to $\mathbb{C P}^{1} \cong S^{2}$ (the variety of one-dimensional subspaces of $\mathbb{C}^{2}$ ) such that, for each $x \in[0,1], a(x) F(x)=$ 0 . Now $a(x)$ has one-dimensional kernel for $x \neq 0$, so necessarily $F(x)=\operatorname{ker} a(x)$ for $x \neq 0$. But the map $(0,1] \rightarrow \mathbb{C P}^{1}$ given by $x \mapsto \operatorname{ker} a(x)$ has no continuous extension $F$ to $[0,1]$, 
since if it did, ker $a(x)$ would have to converge to a limit in $\mathbb{C P}^{1}$, namely $F(0)$, as $x \rightarrow 0_{+}$, and

$$
\lim _{x \rightarrow 0_{+}} \operatorname{ker} a(x)
$$

doesn't exist. One might expect from this that the function $a$ defines a non-zero element of Nil $A$.

Theorem 3.1 runs counter to this expectation. To illustrate some of the main ideas involved, let's show before getting to the general case that any function $a$ from $[0,1]$ to nilpotent $2 \times 2$ matrices, whether or not it can be put in upper-triangular form, represents the trivial element of Nil $A$. Note that the set of nilpotent $2 \times 2$ matrices over $\mathbb{C}$ is

$$
X=\left\{\left(\begin{array}{cc}
x_{1} & x_{2} \\
x_{3} & -x_{1}
\end{array}\right):\left(x_{1}, x_{2}, x_{3}\right) \in \mathbb{C}^{3}, \quad x_{1}^{2}+x_{2} x_{3}=0\right\}
$$

an affine algebraic variety (over $\mathbb{C}$ ). Thus $a:[0,1] \rightarrow X$ induces a map $a^{*}: \mathbb{C}[X] \rightarrow A$, and the element of Nil $A$ defined by $a$ lies in the image of the induced map $\operatorname{Nil} \mathbb{C}[X] \rightarrow \operatorname{Nil} A$. Now there is a proper map of affine varieties $p: \mathbb{C}^{2} \rightarrow X$ defined by

$$
\left(t_{1}, t_{2}\right) \mapsto\left(t_{1} t_{2}, t_{1}^{2},-t_{2}^{2}\right)
$$

which is a "resolution of singularities" for $X$. This map is a branched covering, generically 2-to-1, but branched over the origin 0 (the zero-matrix). Next, observe that any map $a:[0,1] \rightarrow X$ has a factorization

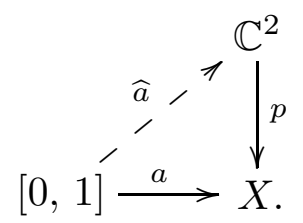

To see this, let $Y=a^{-1}(\mathbf{0})$, which is a closed subset of $[0,1]$. We obtain the lifting by sending $Y$ to $p^{-1}(\mathbf{0})=(0,0) \in \mathbb{C}^{2}$, and mapping the complement of $Y$, which is an open subset of $[0,1]$, hence a countable disjoint union of intervals, using the path lifting property of the covering map $p: \mathbb{C}^{2} \backslash\{(0,0)\} \rightarrow X \backslash\{\mathbf{0}\}$. Then $\widehat{a}:[0,1] \rightarrow \mathbb{C}^{2}$ induces a factorization of the map Nil $\mathbb{C}[X] \rightarrow \operatorname{Nil} A$ through Nil $\mathbb{C}\left[t_{1}, t_{2}\right]$, which is zero.

Before getting to the proof of Theorem 3.1, we need some preliminary results.

3.5. Proposition. Suppose $A$ is a $C^{*}$-algebra (over $\mathbb{F}=\mathbb{R}$ or $\mathbb{C}$ ) and $B$ and $C$ are quotient $C^{*}$-algebras of $A$ (corresponding to closed subsets $\widehat{B}$ and $\widehat{C}$ of the dual space $\widehat{A}$ ). Let $D$ be the quotient of $B$ and $C$ corresponding to the closed set $\widehat{B} \cap \widehat{C}$. Let $r \geq 1$ and let $A[\mathbf{t}]$ be shorthand for $A\left[t_{1}, \ldots, t_{r}\right]$, etc. Then one has a functorial Mayer-Vietoris exact sequence:

$$
\begin{aligned}
\ldots \rightarrow K_{i+1}(D[\mathbf{t}]) \stackrel{\partial}{\longrightarrow} K_{i}(A[\mathbf{t}]) \stackrel{\left(b_{*}, c_{*}\right)}{\longrightarrow} & K_{i}(B[\mathbf{t}]) \oplus K_{i}(C[\mathbf{t}]) \\
\stackrel{d_{1 *}-d_{2 *}}{\longrightarrow} & K_{i}(D[\mathbf{t}]) \stackrel{\partial}{\longrightarrow} K_{i-1}(A[\mathbf{t}]) \rightarrow \ldots
\end{aligned}
$$


Here $b: A[\mathbf{t}] \rightarrow B[\mathbf{t}], c: A[\mathbf{t}] \rightarrow C[\mathbf{t}], d_{1}: B[\mathbf{t}] \rightarrow D[\mathbf{t}], d_{2}: C[\mathbf{t}] \rightarrow D[\mathbf{t}]$ are the quotient maps. The boundary map $\partial$ will be made explicit in the proof. In particular, if the quotient map from $B$ to $D$ splits, then the boundary map $\partial$ vanishes (in all degrees).

Proof. The proof of this is the usual one, granted the excision theorem (1.3). Namely, let $I=\operatorname{ker} d_{1}$ and $J=\operatorname{ker} d_{2}$ (these are ideals of $B[\mathbf{t}]$ and $C[\mathbf{t}]$, respectively), and observe that $I \cong \operatorname{ker} c, J \cong \operatorname{ker} b$. So we have a commutative diagram with exact rows and columns:

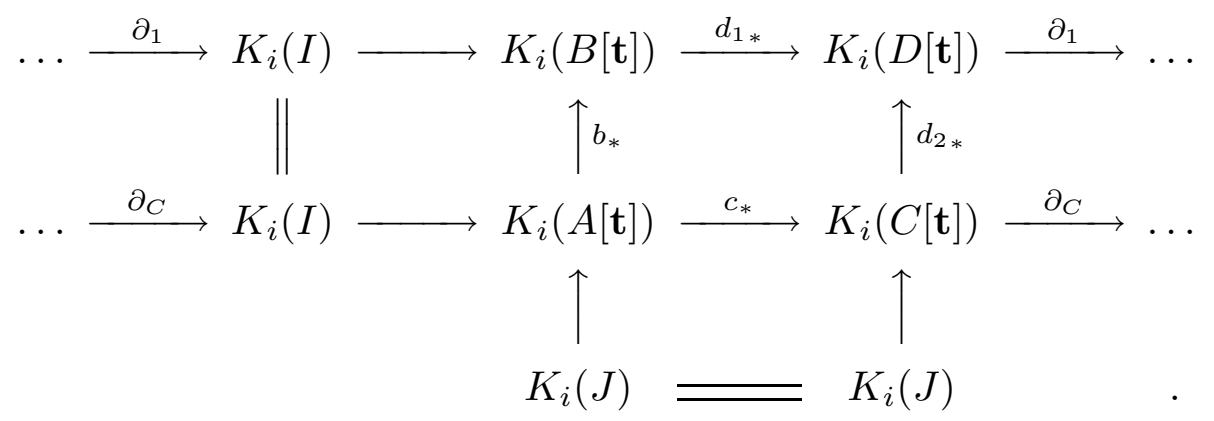

The Mayer-Vietoris exact sequence follows by splicing and diagram-chasing. Note that the boundary map $\partial$ is the "zig-zag" composite

$$
K_{i}(D[\mathbf{t}]) \stackrel{\partial_{1}}{\longrightarrow} K_{i-1}(I) \rightarrow K_{i-1}(A[\mathbf{t}]) .
$$

So if the quotient map $B \rightarrow D$ splits, $d_{1}$ splits, $\partial_{1}$ vanishes, and $\partial$ vanishes.

3.6. Lemma. Let $Y \subseteq X$ be compact Hausdorff spaces and let $Z=X \cup_{Y} c Y$ be the mapping cone of the inclusion $Y \hookrightarrow X$. As usual, denote by $C(X)$ the algebra of continuous complex-valued functions on $X$. Then for any $r \geq 1$, the map

$$
G L\left(C(Z)\left[t_{1}, \ldots, t_{r}\right],\left(t_{1}, \ldots, t_{r}\right)\right) \rightarrow G L\left(C(X)\left[t_{1}, \ldots, t_{r}\right],\left(t_{1}, \ldots, t_{r}\right)\right)
$$

(induced by the restriction map $C(Z) \rightarrow C(X)$ ) is split surjective.

Proof. For simplicity, let's write $A[\mathbf{t}]$ for $A\left[t_{1}, \ldots, t_{r}\right]$. The group

$$
G=G L\left(C(X)[\mathbf{t}],\left(t_{1}, \ldots, t_{r}\right)\right)
$$

is (by definition) the kernel of the map from $G L(C(X)[\mathbf{t}])$ to $G L(C(X))$ induced by sending $t_{i} \mapsto 0$. Similarly, let $H=G L\left(C(Z)[\mathbf{t}],\left(t_{1}, \ldots, t_{r}\right)\right)$. Note that an element of $G$ is a polynomial-valued matrix of the form $1+a(\mathbf{t})$, where the entries of the polynomialvalued matrix $a(\mathbf{t})$ lie in the ideal $\left(t_{1}, \ldots, t_{r}\right)$ and the condition that $1+a(\mathbf{t})$ be invertible is precisely that $a(\mathbf{t})$ should be nilpotent (this guarantees that the power series for the inverse terminates in finitely many steps, hence is polynomial-valued). So we define the splitting map $G \rightarrow H$ as follows:

$$
1+a(\mathbf{t}) \mapsto 1+b(\mathbf{t}),
$$

where $b(t)$ is a polynomial-valued matrix function on $Z$ which agrees with $a(\mathbf{t})$ on $X$, and which on $c Y=Y \times[0,1] / Y \times\{0\}$ is given by

$$
(y, s) \mapsto \begin{cases}a(y)(s \mathbf{t}), & 0<s \leq 1, \\ 0, & s=0 .\end{cases}
$$

Note that this gives a well-defined nilpotent polynomial-valued matrix function on $c Y$ taking the value 0 at the cone point, and agreeing with $a(\mathbf{t})$ on $Y \times\{1\} \cong Y \hookrightarrow Z$. Also note that replacing $\mathbf{t}$ by $s \mathbf{t}$ respects multiplication. So we have the desired splitting. 
3.7. Lemma. Let $Y \subseteq X$ be a finite polyhedral pair. Then for any $r \geq 1$, the boundary map $\partial$ vanishes (in all degrees) in the long exact sequence

$$
\cdots \rightarrow N^{r} K_{i}(C(X)) \rightarrow N^{r} K_{i}(C(Y)) \stackrel{\partial}{\rightarrow} N^{r} K_{i-1}\left(C_{0}(X \backslash Y)\right) \rightarrow \ldots
$$

Proof. Let $Z=X \cup_{Y} c Y$ be as in Lemma 3.6. Then application of the Quillen +construction to the map of groups in Lemma 3.6 shows that the map $N^{r} K_{i}(C(Z)) \rightarrow$ $N^{r} K_{i}(C(X)$ ) is split surjective (for all $i$ ). Similarly (do the same with the inclusion $Y \hookrightarrow X$ replaced by the inclusion $Y \hookrightarrow Y)$, the map $N^{r} K_{i}(C(c Y)) \rightarrow N^{r} K_{i}(C(Y))$ is split surjective (for all $i$ ).

Now use excision in $N^{r} K_{*}$ (Theorem 1.3) to obtain a commutative diagram with exact rows:

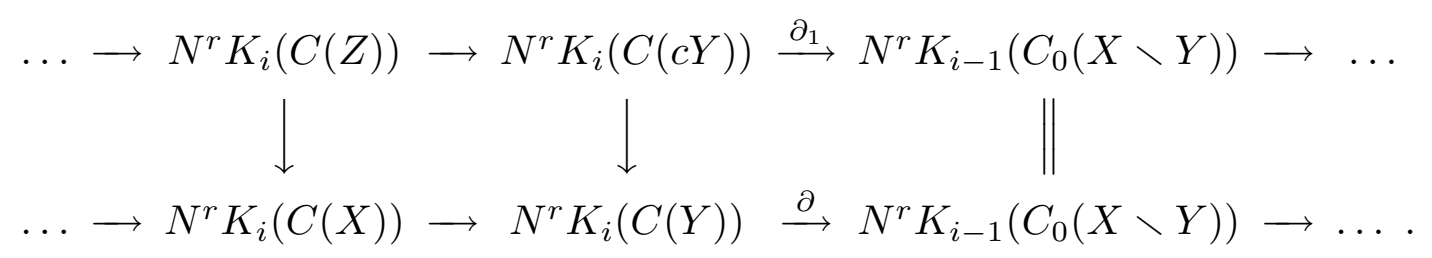

As just pointed out, the first two downward arrows in this diagram are split surjective. But $c Y$ is contractible and $c Y \subseteq Z$ is a polyhedral pair, so $c Y$ is a retract of $Z$ (by simple obstruction theory). Hence the restriction map $C(Z) \rightarrow C(c Y)$ splits and the boundary map $\partial_{1}$ in the first row of the diagram vanishes. Diagram chasing then shows that the boundary map $\partial$ in the second row of the diagram vanishes.

Proof of Theorem 3.1. We need to show that for any $r$ and $i, N^{r} K_{i}(A)=0$. By the excision theorem (1.3), it suffices to consider the case where $A$ is unital. We will just give the proof in the complex case $\mathbb{F}=\mathbb{C}$. Once this is done, the theorem will then follow in the real case $\mathbb{F}=\mathbb{R}$ by a transfer argument, since the fact that $A_{\mathbb{C}}=A \otimes_{\mathbb{R}} \mathbb{C}$ is a free $A$-module of rank 2 implies that the maps $N^{r} K_{i}(A) \rightarrow N^{r} K_{i}\left(A_{\mathbb{C}}\right)$ are injective after inverting 2. Since the $N K$-groups are all complex vector spaces and thus torsion-free [We1], vanishing of $N^{r} K_{i}\left(A_{\mathbb{C}}\right)$ implies vanishing of $N^{r} K_{i}(A)$.

Thus we may assume $A$ is a unital commutative $C^{*}$-algebra over $\mathbb{C}$, hence of the form $C(Y)$ for some compact Hausdorff space $Y$. Next we make an additional reduction to the case where the space $Y$ is a finite polyhedron. Indeed, $A$ is the (algebraic) inductive limit of the filtered family of its finitely generated subalgebras, and since all $K$-functors commute with direct limits, it suffices to fix $r$ and to prove the following:

3.8. Lemma. Let $A$ be a unital commutative $C^{*}$-algebra (over $\mathbb{C}$ ) and let $f_{1}, \ldots, f_{n} \in A$. Then the inclusion into $A$ of $\mathbb{C}\left[f_{1}, \ldots, f_{n}\right]$, the $\mathbb{C}$-subalgebra generated by $f_{1}, \ldots, f_{n}$, induces the zero-map on $N^{r} K_{i}$ for all $i$.

But the algebra $\mathbb{C}\left[f_{1}, \ldots, f_{n}\right]$ is reduced (contains no nilpotent elements), hence by the Nullstellensatz is isomorphic to the algebra $\mathbb{C}[X]$ of regular functions on some affine algebraic set $X \subseteq \mathbb{C}^{N}, N \leq n$, not necessarily irreducible. Then the inclusion $\mathbb{C}\left[f_{1}, \ldots, f_{n}\right] \hookrightarrow$ $A$ is dual to a continuous map $Y \rightarrow X$. The image is a compact subset of the affine algebraic set $X$, which in turn lies in the intersection of $X$ with a closed ball, which is a finite polyhedron $Y^{\prime}$. So the inclusion $\mathbb{C}\left[f_{1}, \ldots, f_{n}\right] \hookrightarrow A$ factors through $C\left(Y^{\prime}\right)$. Thus it suffices to prove the Theorem (and the Lemma) when $Y$ is a finite polyhedron. 
3.9. Sublemma. Suppose $Y$ is a "nice" compact subset of an affine algebraic set $X \subseteq \mathbb{C}^{N}$, and suppose $Y$ is the finite union of "nice" closed subsets $Y_{j}$. Here "nice" means that $Y$ and the $Y_{j}$ are closed finite subpolyhedra for some triangulation of $X$. Assume also that the induced maps $\mathbb{C}[X] \rightarrow C\left(Y_{j}\right)$ have factorizations

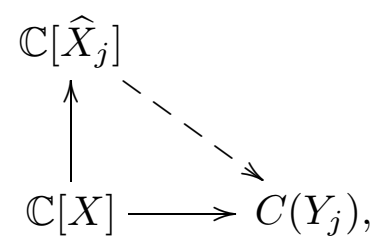

where $\widehat{X}_{j}$ is a nonsingular affine variety. Equivalently, we assume we have factorizations of maps of spaces

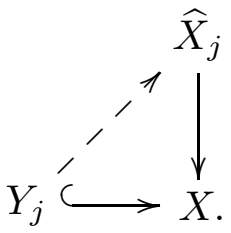

Then the image of $N^{r} K_{i}(\mathbb{C}[X])$ in $N^{r} K_{i}(C(Y))$ vanishes for all $i$.

Proof of Sublemma. We prove this by induction on the number of pieces $Y_{j}$ of $Y$. If there is only one such piece, i.e., $Y=Y_{1}$, then the map from $N^{r} K_{i}\left(\mathbb{C}\left[f_{1}, \ldots, f_{n}\right]\right)$ to $N^{r} K_{i}(C(Y))$ factors through $N^{r} K_{i}$ of the regular ring $\mathbb{C}\left[\widehat{X}_{1}\right]$, which is 0 . To handle the inductive step, we need a Mayer-Vietoris argument. Assume by induction that the image of $N^{r} K_{*}(\mathbb{C}[X])$ in $N^{r} K_{*}(C(Z))$ vanishes whenever $Z=Y_{1} \cup \cdots \cup Y_{m}$ is covered by $m$ closed sets as in the theorem, and then suppose $Y=Y_{1} \cup \cdots \cup Y_{m+1}=Z \cup Y_{m+1}$ is covered by $m+1$ such closed sets. Since we have vanishing of the image of $N^{r} K_{*}(\mathbb{C}[X])$ in $N^{r} K_{*}(C(Z))$ and $N^{r} K_{*}\left(C\left(Y_{m+1}\right)\right)$, Mayer-Vietoris (Proposition 3.5) gives the vanishing in $N^{r} K_{*}(C(Y)$ ) provided the connecting map in the Mayer-Vietoris sequence vanishes. By the description of this connecting map in Proposition 3.5, Lemma 3.7 guarantees the desired vanishing.

Proof of Lemma 3.8. As indicated above, we may suppose $A=C(Y)$ with $Y$ a finite polyhedron. And as also indicated above, we may and do assume the algebra $\mathbb{C}\left[f_{1}, \ldots, f_{n}\right]$ is isomorphic to the algebra $\mathbb{C}[X]$ of regular functions on some affine algebraic set $X \subseteq \mathbb{C}^{N}$, $N \leq n$, and that $Y$ is finite polyhedron embedded as a closed subset of $X$. Write $X$ as a finite union of irreducible affine varieties $X_{j}$. (To simplify notation, we think of affine varieties as point sets in $\mathbb{C}^{N}$ rather than as schemes.) Choose (using [Hir]) a resolution of singularities $p_{j}: \widehat{X}_{j} \rightarrow X_{j}$ for each $j$, so that $\widehat{X}_{j}$ is a smooth irreducible affine variety for each $j$, each projection map $p_{j}: \widehat{X}_{j} \rightarrow X_{j}$ is proper, and the projection map $p_{j}: \widehat{X}_{j} \rightarrow X_{j}$ is one-to-one off the inverse image in $\widehat{X}_{j}$ of a singular set in $X_{j}$ which is a subvariety of lower dimension. (We won't really need the condition that $p_{j}$ is generically one-to-one; it would suffice for it to be a submersion off the singular set, as in Example 3.4.) We may assume as in the Sublemma that $Y$ is a "nice" compact subset of $X$. If we can find a covering of $Y \cap X_{j}$ for each $j$ by closed subsets with liftings to $\widehat{X}_{j}$ as in the Sublemma, then we will have satisfied the condition of the Sublemma for a closed covering of $Y$ (the union of all these coverings), and will have thus proved Lemma 3.8. So without loss of generality, we may assume $X$ irreducible with a resolution $p: \widehat{X} \rightarrow X$. As explained in [GoM], it 
is possible to find a Whitney stratification of $X$ compatible with $p$. In other words, $X$ is partioned into a finite union of locally closed subsets, called the pure strata $X^{\alpha}, \alpha$ ranging over a finite partially ordered set, so that each $X^{\alpha}$ is a smooth (complex) manifold, the projection map $p: \widehat{X} \rightarrow X$ is a proper submersion, hence a fibration, when restricted to $\widehat{X}^{\alpha}=p^{-1}\left(X^{\alpha}\right)$, and such that there is a neighborhood $T^{\alpha}$ of $X^{\alpha}$ in $X$ with a locally trivial projection $T^{\alpha} \rightarrow X^{\alpha}$ with fibers homeomorphic to the cone $c L^{\alpha}$ on some finite polyhedron $L^{\alpha}$ (called the link of the stratum) [GoM, p. 41]. (In Example 3.4, one may take the two strata of $X$ to be $\{\mathbf{0}\}$ and its complement. The link of the singular stratum is $\mathbb{R P}^{3}$.) We need to construct a covering of $Y$ by closed sets with liftings to the "desingularization" $\widehat{X}$ as in the Sublemma. (In principle we could use different desingularizations for the different sets of the covering, but this turns out to be unnecessary.) We use the fact that $X$ has a closed covering by sets of the form $D^{\alpha} \times c L^{\alpha}$, where $D^{\alpha}$ is a closed disk in $X^{\alpha}$ (the closure of a small coordinate chart in $\left.X^{\alpha}\right)$. Since $p: \widehat{X}^{\alpha} \rightarrow X^{\alpha}$ is a fibration, $p$ is trivial over the contractible set $D^{\alpha} \subset X^{\alpha}$. Let $\widehat{L}^{\alpha}=p^{-1}\left(L^{\alpha}\right)$, which is the link of $\widehat{X}^{\alpha}$ in $\widehat{X}$. (Note, incidentally, that since $\widehat{X}$ is smooth, $\widehat{L}^{\alpha}$, the link of $\widehat{X}^{\alpha}$ in $\widehat{X}$, is topologically a sphere.) The link $L^{\alpha}$ is stratified by its intersections with the other strata, and note that the maximum number of strata of $L^{\alpha}$ is less than the number of strata of $X$. (For example, if $X$ has only two strata, the top stratum is open, and the link of the singular stratum is a manifold.) The map $p$ gives a stratified surjection $\widehat{L}^{\alpha} \rightarrow L^{\alpha}$ compatible with the cone structure on a neighborhood of $X^{\alpha}$ (resp., $\widehat{X}^{\alpha}$ ). By an argument using induction on the number of strata, each $L^{\alpha}$ can be covered by finitely many closed sets $W$ over which the map $\widehat{L}^{\alpha} \rightarrow L^{\alpha}$ becomes trivial. (For example, if $L^{\alpha}$ is non-singular, $p$ gives a fibration $\widehat{L}^{\alpha} \rightarrow L^{\alpha}$, which becomes trivial over a family of contractible subsets of $L^{\alpha}$, e.g., the closed simplices for some triangulation, and the map $p: \widehat{X} \rightarrow X$ takes the local form

$$
p^{-1}\left(D^{\alpha}\right) \times c p^{-1}(W) \rightarrow D^{\alpha} \times c W,
$$

where $p: p^{-1}\left(D^{\alpha}\right) \rightarrow D^{\alpha}$ and $p: p^{-1}(W) \rightarrow W$ are trivial fibrations, i.e., projections onto one factor in a product. Thus there is a continuous section $D^{\alpha} \times c W \rightarrow p^{-1}\left(D^{\alpha} \times c W\right)$ which gives a lifting of the desired type on $\left(D^{\alpha} \times c W\right) \cap Y$. If $L^{\alpha}$ is singular, we iterate this process by looking at tubular neighborhoods of the strata of $L^{\alpha}$, getting a local structure for $p$ of the form

$$
p^{-1}\left(D^{\alpha}\right) \times c p^{-1}(W) \rightarrow D^{\alpha} \times c W
$$

with $W$ itself a product of a disk and a cone, etc.) By compactness of $Y, Y$ has a finite covering by sets of this form, and we are done.

3.10. Theorem. Let $B$ be a type $I C^{*}$-algebra (over $\mathbb{F}=\mathbb{R}$ or $\mathbb{C}$ ) which has a finite composition series, each of whose composition factors has the form $A \otimes M_{n}(\mathbb{F})$ ( $n \geq 0$ ) or $A \otimes \mathcal{K}$, where $A$ is commutative. Then $B$ is $K_{i}$-regular for all $i$.

Proof. We argue by induction on the number of composition factors. To start the induction, observe that the case $B=A \otimes \mathcal{K}$ is covered by Theorem 1.5 , and the case $B=A \otimes M_{n}$ is covered by Theorem 3.1, together with Morita invariance of algebraic $K$-theory. For the inductive step, assume we know the result for algebras $B^{\prime}$ and $B^{\prime \prime}$ and consider a $C^{*}$-algebra $B$ with a short exact sequence

$$
0 \rightarrow B^{\prime} \rightarrow B \rightarrow B^{\prime \prime} \rightarrow 0
$$


Let $r \geq 1$, let $B[\mathbf{t}]$ be shorthand for $B\left[t_{1}, \ldots, t_{r}\right]$, and consider the commutative diagram with exact rows:

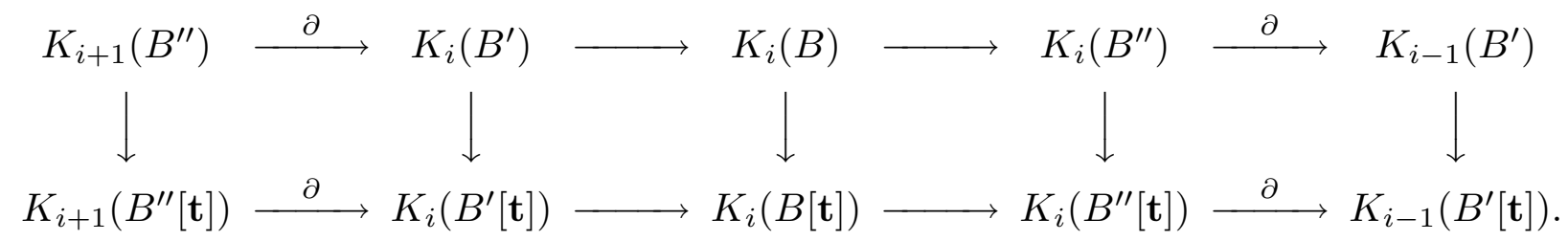

By inductive hypothesis, the four outer maps are isomorphisms, so by the Five-Lemma, so is the inner map.

3.11. Corollary. If $B$ is a type $I C^{*}$-algebra as in Theorem 3.10, then the Bass-Quillen $K$-groups of $B, K_{i}(B)$; the Karoubi-Villamayor $K$-groups of $B, K V_{i}(B)$; and the Weibel homotopy algebraic $K$-groups of $B, K H_{i}(B)$, are all naturally isomorphic to one another.

Proof. This is an immediate consequence of $K_{i}$-regularity for all $i$-see [We2, Proposition $1.5]$.

\section{Higher Algebraic $K$-TheOrY AND ANALOGUES OF THE Fischer-PRASOlOV ThEOREM}

Theorem 1.7 suggests a somewhat audacious conjecture, for which the evidence is admittedly somewhat flimsy.

4.1. Conjecture. Let $A$ be any $C^{*}$-algebra. Then the map of Theorem 1.1 induces isomorphisms of $K$-groups with finite coefficients

$$
K_{i}(A ; \mathbb{Z} / k) \rightarrow K_{i}^{\text {top }}(A ; \mathbb{Z} / k), \quad i \geq 1
$$

for any $k>1$.

This conjecture is rather easy to check for $i=1$, and for $i>1$, it's already known that the indicated map is surjective. Both of these results are in [K, Théorème 2.5]. The best evidence for the conjecture lies in the fact that it is true in two "opposite" cases, when $A$ is commutative (Theorem 1.7) and when $A$ is stable (Theorem 1.4). Putting these two cases together gives the result for many type $\mathrm{I} C^{*}$-algebras.

4.2. Theorem. Let $B$ be a type $I C^{*}$-algebra (over $\mathbb{F}=\mathbb{R}$ or $\mathbb{C}$ ) which has a finite composition series, each of whose composition factors has the form $A \otimes M_{n}(\mathbb{F})(n \geq 0)$ or $A \otimes \mathcal{K}$, where $A$ is commutative. Then Conjecture 4.1 holds for $B$.

Proof. The proof is quite similar to that of Theorem 3.10. We argue by induction on the number of composition factors. To start the induction, observe that the case $B=A \otimes \mathcal{K}$ is covered by Theorem 1.4, and the case $B=A \otimes M_{n}$ is covered by Theorem 1.7, together with Morita invariance of algebraic $K$-theory. However, we will later need one additional fact, which is slightly more subtle, namely that in both cases the map $K_{0}(A ; \mathbb{Z} / k) \rightarrow$ $K_{0}^{\text {top }}(A ; \mathbb{Z} / k)$ is also an isomorphism. (The problem here is that $K_{0}(A ; \mathbb{Z} / k)$ surjects onto the $k$-torsion in $K_{-1}$, whereas negative algebraic and topological $K$-theory do not always coincide.) The fact that the map $K_{0}(A ; \mathbb{Z} / k) \rightarrow K_{0}^{\text {top }}(A ; \mathbb{Z} / k)$ is an isomorphism is again part of Theorem 1.4 in the stable case, but in the abelian case, it requires Theorem 2.3, 
together with the fact that on finite $\mathrm{CW}$-complexes, $k u^{1}$ and $K U^{1}$ coincide, and similarly for $k O^{1}$ and $K O^{1}$. (This can be seen from examination of the Atiyah-Hirzebruch spectral sequences.) For the inductive step, assume we know the result for algebras $B^{\prime}$ and $B^{\prime \prime}$ and consider a $C^{*}$-algebra $B$ with a short exact sequence

$$
0 \rightarrow B^{\prime} \rightarrow B \rightarrow B^{\prime \prime} \rightarrow 0,
$$

with $B^{\prime}$ of the form $A \otimes \mathcal{K}$ or $A \otimes M_{n}, A$ abelian. Consider the commutative diagram with exact rows:

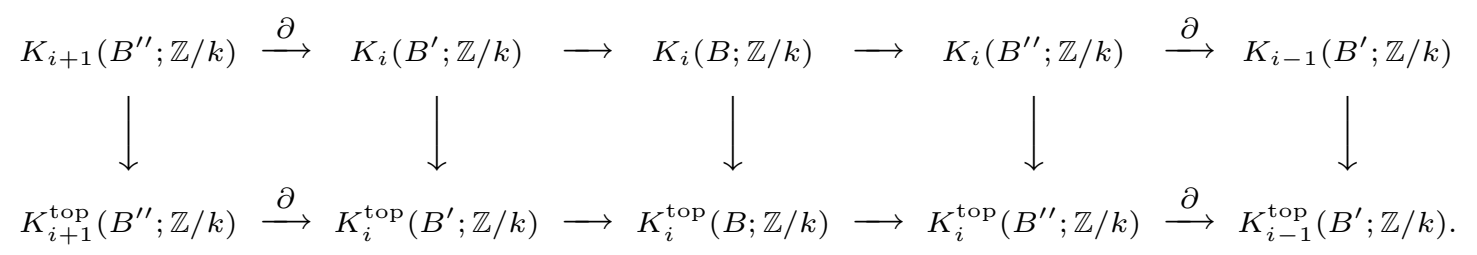

By inductive hypothesis (plus what we know about $B^{\prime}$ in degree 0 ), the four outer maps are isomorphisms, so by the Five-Lemma, so is the inner map.

Theorem 4.2 suggests that the best test for Conjecture 4.1 should be a UHF algebra, the $C^{*}$-algebraic inductive limit of a sequence of matrix algebras and unital maps, or more generally an AF algebra, the $C^{*}$-algebraic inductive limit of a sequence of finitedimensional algebras, or still more generally, an inductive limit of type I $C^{*}$-algebras which are direct sums of tensor products of abelian algebras with matrix algebras. Recent developments in $C^{*}$-algebra theory show that such inductive limits are quite a large class of $C^{*}$-algebras, and include many of the best-known simple nuclear algebras. The best result we have been able to prove about these is the following. Note that this is only a very modest improvement over what is already proved in [K, Théorème 2.5] for general Banach algebras, the difference being the construction of an explicit splitting map.

4.3. Theorem. Let $A$ be an $A F$ algebra over $\mathbb{F}=\mathbb{R}$ or $\mathbb{C}$, or more generally an inductive limit of type $I C^{*}$-algebras which are direct sums of tensor products of abelian algebras with matrix algebras. Then the map of Theorem 1.1 induces split surjections of $K$-groups with finite coefficients

$$
K_{i}(A ; \mathbb{Z} / k) \rightarrow K_{i}^{\mathrm{top}}(A ; \mathbb{Z} / k), \quad i \geq 1
$$

for any $k>1$.

Proof. By excision, it is no loss of generality to assume $A$ is unital and the maps in the inductive limit are unital. Write $A$ as an inductive limit of an increasing sequence $B_{j}$ of finite-dimensional algebras (in the AF case) or of direct sums of tensor products of abelian algebras with matrix algebras, and let $A_{0} \subset A$ be the algebraic inductive limit of the sequence. (So $A_{0}$ is a dense subalgebra of $A$. In the AF algebra case, $A_{0}$ has countable dimension over $\mathbb{C}$.) By continuity of algebraic and topological $K$-theory with respect to algebraic and topological direct limits, respectively, we have $K_{i}\left(A_{0} ; \mathbb{Z} / k\right)=\varliminf_{i} K_{i}\left(B_{j} ; \mathbb{Z} / k\right)$ and $K_{i}^{\mathrm{top}}(A ; \mathbb{Z} / k)=\varliminf_{i} K_{i}^{\mathrm{top}}\left(B_{j} ; \mathbb{Z} / k\right)$. But the comparison map $K_{i}\left(B_{j} ; \mathbb{Z} / k\right) \rightarrow K_{i}^{\mathrm{top}}\left(B_{j} ; \mathbb{Z} / k\right)$ is an isomorphism by Suslin's Theorem or the Fischer-Prasolov Theorem (Theorem 1.7) and Morita invariance. So the inclusion $A_{0} \hookrightarrow A$ induces a splitting map

$K_{i}^{\text {top }}(A ; \mathbb{Z} / k)=\varliminf_{\longrightarrow} K_{i}^{\text {top }}\left(B_{j} ; \mathbb{Z} / k\right) \cong \varliminf_{\lim } K_{i}\left(B_{j} ; \mathbb{Z} / k\right)=K_{i}\left(A_{0} ; \mathbb{Z} / k\right) \rightarrow K_{i}(A ; \mathbb{Z} / k)$.

We also have a quite parallel result for transformation group $C^{*}$-algebras, that is, crossed products of a commutative $C^{*}$-algebra by an action of $\mathbb{Z}$. 
4.4. Theorem. Let $A=B \rtimes_{\alpha} \mathbb{Z}$ be a crossed product of a commutative $C^{*}$-algebra $B$ (over $\mathbb{F}=\mathbb{R}$ or $\mathbb{C}$ ) by an action $\alpha$ of $\mathbb{Z}$. Then the map of Theorem 1.1 induces split surjections of $K$-groups with finite coefficients

$$
K_{i}(A ; \mathbb{Z} / k) \rightarrow K_{i}^{\mathrm{top}}(A ; \mathbb{Z} / k), \quad i \geq 1,
$$

for any $k>1$.

Proof. By excision, it is no loss of generality to assume $B$ (and hence $A$ ) is unital. Let $A_{0}$ be the algebraic crossed product, sometimes denoted $B_{\alpha}\left[x, x^{-1}\right]$. This is the dense subalgebra of $A$ spanned (algebraically) by $B$ and by the unitary element $x$ corresponding to the generator of $\mathbb{Z}$. Now the topological $K$-theory of $A$ is computed by the PimsnerVoiculescu exact sequence [PV], whereas the algebraic $K$-theory of $A_{0}$ is computed by an algebraic counterpart to this exact sequence, basically due to Grayson [Gr] (but see also [Ran], pages 427-428, for an explanation of why Grayson's localization theorem is applicable). The algebraic case differs from the topological one in the presence of "Nil" terms, but these disappear when working with finite coefficients (for the same reasons in the $\alpha$-twisted case as in the untwisted case discussed in [We1]). Putting everything together, we get a commutative diagram with exact rows:

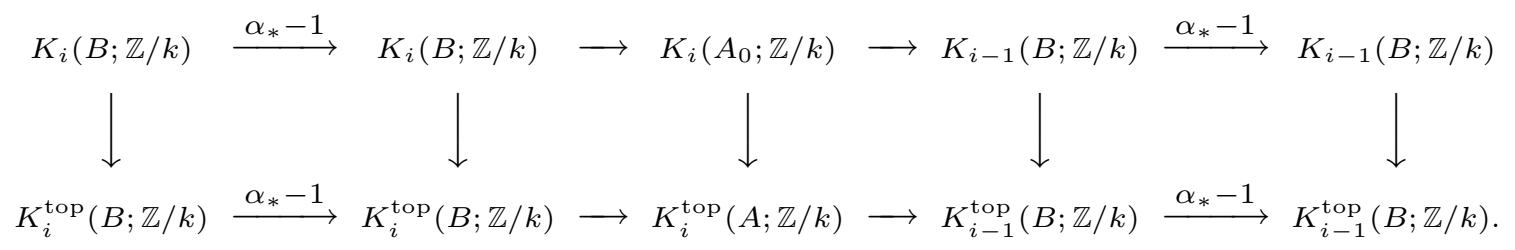

By an application of the 5-Lemma, just as in Theorem 4.3, we find that $K_{i}\left(A_{0} ; \mathbb{Z} / k\right) \rightarrow$ $K_{i}^{\text {top }}(A ; \mathbb{Z} / k)$ is an isomorphism. So the inclusion $A_{0} \hookrightarrow A$ induces a splitting map

$$
K_{i}^{\mathrm{top}}(A ; \mathbb{Z} / k) \cong K_{i}\left(A_{0} ; \mathbb{Z} / k\right) \rightarrow K_{i}(A ; \mathbb{Z} / k) .
$$

\section{Some Results on Bass Stable Rank}

The results of this section are worked out for the polynomial ring in one variable over $C([0,1])$, but presumably one could with more work but with similar techniques study polynomial rings in more variables or over more complicated commutative $C^{*}$-algebras.

5.1. Theorem. Let $A=C^{\mathbb{C}}([0,1])$ and let $B=A[t]$. Then $B$ has stable rank exactly 2 (in the sense of Bass).

Proof. The ring $B$ cannot have stable rank 1, for the same reason as $\mathbb{C}[t]$ (the functions $t$ and $1+t^{2}$ generate $B$ as an ideal, but no sum of $t$ and a multiple of $1+t^{2}$ is invertible). So it's enough to show $B$ has stable rank $\leq 2$.

First note that $B$, an overring of the topological ring $A$, is a topological ring for its inductive limit topology as an inductive limit of finitely generated free $A$-modules, so its maximal ideals must be closed in this topology. In particular, if $I$ is a maximal ideal in $B$, then $I \cap A$ is a closed prime ideal in $A$, hence consists of the functions vanishing at some point $x \in[0,1]$, and $B / I$ is an algebraic field extension of $A / I \cap A \cong \mathbb{C}$, hence (since $\mathbb{C}$ is algebraically closed) $B / I=A / I \cap A$. Thus $I$ must consist of those elements vanishing 
at some uniquely defined pair $x \in[0,1], t \in \mathbb{C}$. Conversely, the functions vanishing at such a pair are clearly a maximal ideal, so the maximal ideals of $B$ are parameterized by $[0,1] \times \mathbb{C}$. A collection of elements of $B$ generate $B$ (algebraically) as an ideal if and only if they are not contained in a maximal ideal, i.e., they have no common zeros when viewed as functions on $[0,1] \times \mathbb{C}$.

Now suppose $a_{1}, a_{2}, a_{3} \in B$ generate $B$ as an ideal. This means that as functions on $[0,1] \times \mathbb{C}$, they have no common zeros. We need to show that there exist $b_{1}, b_{2} \in B$ such that $a_{1}+b_{1} a_{3}, a_{2}+b_{2} a_{3}$ generate $B$ as an ideal. In fact, we can take $b_{1}(x, t)$ and $b_{2}(x, t)$ to be independent of $t$. To see this, define for each $x \in[0,1]$ a subset $G(x)$ of $\mathbb{C} \times \mathbb{C}$ by

$$
\begin{aligned}
G(x)=\left\{\left(\lambda_{1}, \lambda_{2}\right) \in\right. & \mathbb{C} \times \mathbb{C}: a_{1}(x, t)+\lambda_{1} a_{3}(x, t), \\
& \left.\left.a_{2}(x, t)+\lambda_{2} a_{3}(x, t) \text { have no common zero (in } t\right)\right\} .
\end{aligned}
$$

Since, by assumption, $a_{1}(x, t), a_{2}(x, t)$, and $a_{3}(x, t)$ have no common zero (in $t$, for $x$ fixed), this set is non-empty. In fact, its complement in $\mathbb{C} \times \mathbb{C}$ is either empty (if $a_{3}(x, t) \equiv 0$ ), or finite (if for example $a_{1}(x, t) \equiv a_{2}(x, t) \equiv 0$ ), or a divisor (the vanishing set of some resultant). In any event, since one can get bounds (in $x$ ) on the coefficients of the resultant polynomials defining the $G(x)^{\text {'s, }}$, it is then easy to see that there is a continuous selection function

$$
\left(b_{1}, b_{2}\right):[0,1] \rightarrow \mathbb{C} \times \mathbb{C}, \quad\left(b_{1}(x), b_{2}(x)\right) \in G(x)
$$

Retain the notations $A=C^{\mathbb{C}}([0,1])$ and $B=A[t]$. We have $S K_{1}(A)=0$ since $A$ has stable rank 1. Since, in addition, $A$ has no non-zero nilpotents, $B^{\times}=A^{\times}$and Nil $A=$ $S K_{1}(B)=S K_{1}(B, I)$, where $I$ is the ideal in $B$ generated by $t$. By Theorem $5.1, B$ has stable rank 2, so $S K_{1}(B, I)$ is generated by Mennicke symbols (for the elementary theory of these, see [Ro2, proof of Theorem 2.3.11 and Theorem 2.5.12]), and in fact by [Va2, Theorem 6], the standard relations among Mennicke symbols (as listed in [Ro2, Theorem 2.5.12] and [Va2]) give a presentation of $S K_{1}(B, I)$. In other words, Nil $A$ is the free abelian group on symbols $[1+t f(x, t) \quad t g(x, t)], f, g \in B$ with $1+t f$ and $t g$ having no common zeros $($ in $[0,1] \times \mathbb{C})$, subject to the relations:

(5.2a) $[1+t f \quad t g]=[1+t f \quad t g+t h(1+t f)]$

$(5.2 \mathrm{~b})\left[\begin{array}{ll}1+t f & t g\end{array}\right]=\left[\begin{array}{ll}1+t f+t g h & t g\end{array}\right]$

(5.3c) $\left[1+t f \quad t^{2} g h\right]=\left[\begin{array}{ll}1+t f & t g\end{array}\right]\left[\begin{array}{ll}1+t f & t h\end{array}\right]$,

whenever these make sense. (As pointed out in the introduction of [Va2], these imply the other well-known relations, such as the fact that $\left[\begin{array}{ll}1 & t g\end{array}\right]=1$.) Theorem 3.1 (or Example 3.4) gives the somewhat unexpected fact that this presentation defines the trivial group, a fact which seems quite hard to show directly.

\section{REFERENCES}

[Ad] J. F. Adams, Infinite Loop Spaces, Annals of Math. Studies, vol. 90, Princeton Univ. Press, Princeton, NJ, 1978.

[F] T. Fischer, K-theory of function rings, J. Pure Appl. Alg. 69 (1990), 33-50.

[Ger] S. Gersten, On the spectrum of algebraic K-theory, Bull. Amer. Math. Soc. 78 (1972), $216-219$.

[GoM] M. Goresky and R. MacPherson, Stratified Morse Theory, Ergebnisse der Math. und Grenzgebiete, 3. Folge, vol. 14, Springer-Verlag, Berlin, New York, 1988. 
[Gr] D. Grayson, K-theory and localization of noncommutative rings, J. Pure and Appl. Alg. 18 (1980), 125-127.

[Hig] N. Higson, Algebraic K-theory of stable $C^{*}$-algebras, Adv. in Math. 67 (1988), 1-140.

[Hir] H. Hironaka, Resolution of singularities of an algebraic variety over a field of characteristic zero, I, II, Ann. of Math. 79 (1964), 109-203, 205-326.

[K] M. Karoubi (with an appendix by W. van der Kallen), Homologie de groupes discrets associés à des algèbres d'opérateurs, J. Operator Theory 15 (1986), 109-161.

[OP] C. Olsen and G. K. Pedersen, Corona $C^{*}$-algebras and their applications to lifting problems, Math. Scand. 64 (1989), 63-86.

[PV] M. Pimsner and D. Voiculescu, Exact sequences for K-groups and Ext-groups of certain crossproducts of $C^{*}$-algebras, J. Operator Theory 4 (1980), 93-118.

[Pr] A. V. Prasolov, Algebraic K-theory of Banach algebras, Dokl. Akad. Nauk B.S.S.R. 28 (1984), 677-679; English translation, Selected Papers in K-theory, Amer. Math. Soc. Translations, Ser. 2, vol. 154, Amer. Math. Soc., Providence, RI, 1992.

[Ran] A. Ranicki, Exact sequences in the algebraic theory of surgery, Mathematical Notes, vol. 26, Princeton Univ. Press, Princeton, NJ, 1981.

[Ro1] J. Rosenberg, $K$ and $K K$ : topology and operator algebras, Operator Theory / Operator Algebras and Applications, Proc. Symp. Pure Math., vol. 51, Part 1, Amer. Math. Soc., Providence, RI, 1990, pp. 445-480.

[Ro2] J. Rosenberg, Algebraic K-Theory and its Applications, Graduate Texts in Math., vol. 147, Springer-Verlag, New York, Berlin, 1994.

[S] A. A. Suslin, On the K-theory of local fields, J. Pure Appl. Alg. 34 (1984), 301-318.

[SW] A. A. Suslin and M. Wodzicki, Excision in algebraic K-theory, Ann. of Math. 136 (1992), 51-122.

[Swa] R. Swan, On seminormality, J. Algebra 67 (1980), 210-229.

[Va1] L. N. Vaserstein, Bass's first stable range condition, J. Pure Appl. Alg. 34 (1984), 319-330.

[Va2] L. N. Vaserstein, Computation of $K_{1}$ via Mennicke symbols, Comm. Alg. 15 (1987), 611-656.

[Vo] T. Vorst (with an appendix by W. van der Kallen), Localization of the K-theory of polynomial extensions, Math. Ann. 244 (1979), 33-53.

[Wag] J. B. Wagoner, Delooping classifying spaces in algebraic K-theory, Topology 11 (1972), 349-370.

[We1] C. Weibel, Mayer-Vietoris sequences and module structures on $N K_{*}$, Algebraic $K$-Theory, Proceedings, Evanston, 1980 (E. M. Friedlander and M. R. Stein, eds.), Lect. Notes in Math., vol. 854, Springer-Verlag, Berlin, New York, 1981, pp. 466-493.

[We2] C. Weibel, Homotopy algebraic K-theory, Algebraic K-Theory and Algebraic Number Theory, Proceedings, Honolulu, 1987 (R. K. Dennis and M. R. Stein, eds.), Contemp. Math., vol. 83, Amer. Math. Soc., Providence, RI, 1989, pp. 461-488.

[Wo] M. Wodzicki, Excision in cyclic homology and in rational algebraic K-theory, Ann. of Math. 129 (1989), 591-639.

Department of Mathematics, University of Maryland, College Park, MD 20742

E-mail address: jmr@math.umd.edu 